\title{
Champagne Bubble Test: An Authentic Method to Find Hard-to-Find Canals during Root Canal Therapy
}

\section{Farid A Faruqi ${ }^{1}$, Asaad J Mirza ${ }^{2 *}$ and Rafique Moosa $^{3}$}

${ }^{1}$ Department of Operative Dentistry, Dow University of Health Sciences, Pakistan

${ }^{2}$ Department of Operative Dentistry, Baqai Medical University, Pakistan

${ }^{3}$ Department of Prosthodontics, Baqai Medical University, Pakistan

*Corresponding Author: Asaad J Mirza, Department of Operative Dentistry, Baqai

Medical University, Pakistan.
Received: August 25, 2021

Published: September 23, 2021

(C) All rights are reserved by Asaad J Mirza., et al.

\begin{abstract}
During an endodontic treatment, a dentist encounters multiple variations in the configuration of the root canal system. This morphologic variation makes many canals very difficult to find and the operating dentist has to take various measures to locate such hard-to-find canals. Champagne bubble test is a method which helps to pinpoint orifice of a hidden canal. This case report describes a similar problem rectified with the help of champagne bubble test.

In mandibular first molars, presence of middle mesial (MML) canal with low incidence has been documented. In Pakistan, its existence has been reported as low as 3.3\% which may be missed by a dentist because of various reasons including its rare occurrence, reparative dentin deposition and lack of dentist's patience, skill and application of proper protocol for discovering hidden canals. Leaving such canals unexplored and consequently untreated, leads to failure of the whole endodontic procedure. Caries was removed in tooth \# 46 and located canals; Mesiobuccal (MB), Mesiolingual (ML), Distobuccal (DB) and distolingual (DL) were accessed, working length established and were instrumented under copious irrigation. Broader buccolingual dimension of the tooth and long span between MB and ML canals were a clue for possibility of another canal in the mesial root. Despite applying all available diagnostic measures the suspected missing canal couldn't be located. Champagne bubble test, however, succeeded to locate it.
\end{abstract}

Keywords: Champagne Bubble Test; Hidden Root Canals; Hard-to Find-Canals; Mesial Middle Canal

\section{Abbreviations}

RCT: Root Canal Therapy; MML: Middle Mesial Canal; MB: Mesiobuccal; ML: Mesiolingual; DB: Distobuccal; DL: Distolingual; NaO$\mathrm{Cl}$ : Sodium Hypochlorite

\section{Introduction}

Mandibular first molars usually have two roots with three canals; two canals in the mesial and one canal in the distal root. Their root canal system is complex with widely found isthmii and anatomical variations [1]. The existence of a third canal in the mesial root has been reported in various studies and its incidence in Pakistani population has been reported merely 3.3\% [2]. Similarly, presence of $2^{\text {nd }}$ canal in a distal root in Indian population which has many similarities in climate and culture with Pakistani population, has been reported around 5\% [3]. As such variations are rare, the clinician for positive outcome of his/her clinical effort must possess thorough knowledge and should be more focused and atten- 
tive during access preparation to be able to locate all canal orifices present in the pulp chamber.

The access to the pulp chamber should be prepared to a size allowing removal of all soft and hard tissue and restorative material. The chamber should be carefully inspected for tissue remnants, overhangs, dentin stones, presence of reparative dentine and root canal orifices under copious irrigation with $\mathrm{NaOCl}$. Mechanical debridement of the canals should be delayed until preparation and cleaning of the chamber has been thoroughly accomplished and a strict protocol has been followed during the search for hidden or hard -to- find canal orifices [4].

\section{History and Treatment}

A 24-year old male patient attended our clinic with complaint of sharp pain on the right side of his face. The pain was acute and used to radiate towards temple and ear on the same side. He was using Tab. Flurbiprofen $100 \mathrm{mg}$ (Ansaid; Pfizer Inc, New York) for around a month to kill the pain. He, otherwise, was medically fit young student with habit of frequent beverage consumption. Intraoral examination revealed gross caries in tooth \# 46 but other teeth were caries free. Periapical radiograph showed caries approaching the distal pulp horn of the affected tooth (Picture 1). Long lasting painful response from thermal and electrical pulp testing led to diagnosis of "symptomatic irreversible pulpitis." Gentle manual removal of caries exposed the involved pulp horn leaving extraction or Root Canal Treatment (RCT) as a possible solution to the problem.

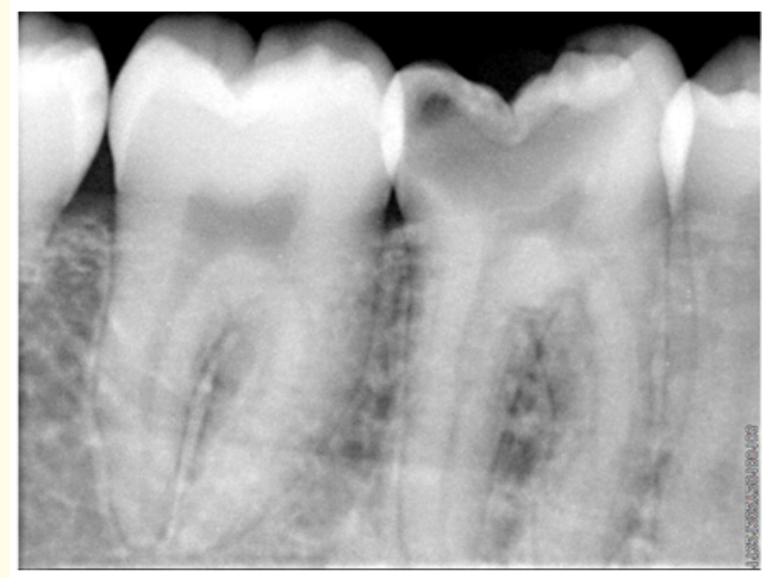

Picture 1: Caries approaching distal pulp horn.
The patient accepted the option to undergo RCT and Having his consent, chamber of the tooth was opened under mandibular anesthesia. For visualization of canal orifices, dentinal shelves were removed with Endo Z carbide bur having non-cutting safety tip mounted on High Speed turbine (Dentsply USA) and patency file $\mathrm{K}$ \# 10 (Shanghai Luk Co. China) was passively inserted into all visible canals for confirmation of their existence. Using Gates Glidden burs in step-back order in the sequence of 2, 3 and 4, straight line access was gained in MB, ML, DB and DL canals and working lengths were measured using an electronic apex locator (Root ZX; J Morita Corporation). For coronal $3^{\text {rd }}$ preparation, Protaper $\mathrm{S}_{\mathrm{x}}$ (Shanghai Luk Co. China) was used in all canals. MB and ML canals were finished using 25/06 SkyTaper system (Komet Dental; Trophagener Weg, Lemgo, Germany) whereas DB and DL were prepared with 35/06. Komet-skytaper is a single file system with .06 taper. Throughout the debridement, copious irrigation was performed using 5\% Sodium Hypochlorite $(\mathrm{NaOCl})$ and $17 \%$ Ethylenediaminetetraacetic acid (EDTA) gel.

The distal root generally has one canal but two canals, DB and DL existed in this case, and broader buccolingual dimension of tooth \#46 and long span between MB and ML canals was indicative of presence of another canal in the mesial root.

It was decided to look for the probably missing canal which was not very evident on the radiograph. To enhance visibility of the chamber floor, 17\% EDTA solution and 95\% ethanol was sequentially applied for better cleaning and drying of the pulp chamber. Ultrasonic scaler tips were employed for further cleaning and deepening the canal isthmii but the third mesial canal could not be located. Finally, "champagne bubble" test which is quite easy to apply and requires no additional instrument, was employed to discover the hidden orifice. The chamber was filled with $\mathrm{NaOCl}$ and left standing for few moments. Under magnification using pair of loupes capable of magnifying an object ranging from $2.5 \mathrm{x}$ to $3.5 \mathrm{x}$, tiny bubbles were visible in the solution indicating the position of the missing canal orifice. DG 16, used with moderate finger pressure at the site of bubbles, confirmed the presence of middle mesial canal (MML) which originated as a separate orifice near ML canal (Picture 2) but joined apically. It was initially negotiated with file $\mathrm{K}$ \#8 and was prepared with 25/04 Komet F360 rotary system.

On the next appointment, the patient was symptomless and the prepared canals were effortlessly dried and found ready for obturation (Picture 3). 


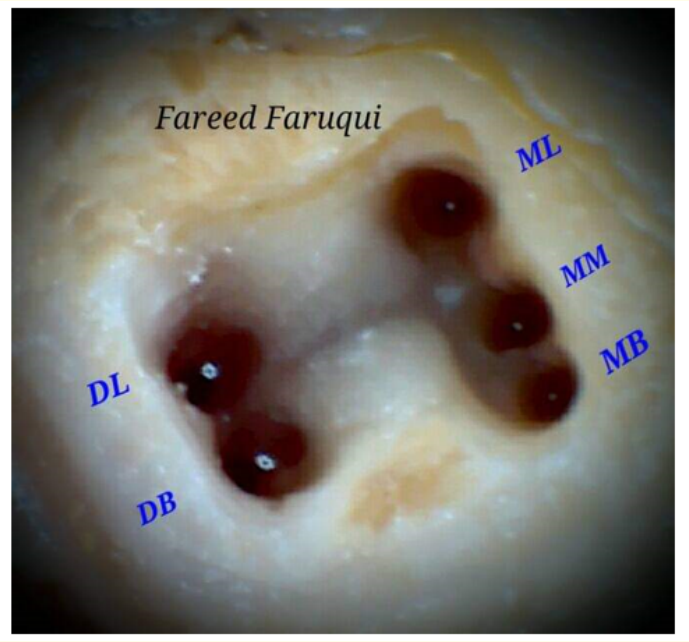

Picture 2: MM canal present in tooth \#46.

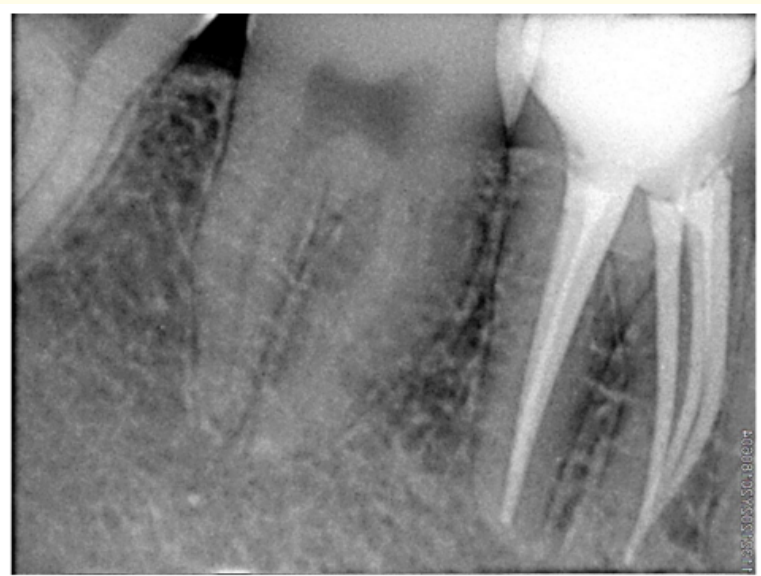

Picture 3: Three Mesial canals obturated.

\section{Discussion}

Many times, dentists fail to find all the canal orifices present in the chamber due to various reasons which culminates into an unsuccessful RCT [5]. In this clinical case, there was tertiary dentin deposition making it difficult to find its canals. Generally, mandibular first molar has MB, ML canals and one centrally located, larger distal canal. While performing RCT, the operator should keep potential anatomic variations in mind for every tooth but mandibular molars have comparatively consistent root canal anatomies [6]. There are documented clinical cases reporting five, six or more canals in the molars. A radiographic study on extracted first lower molars has reported incidence of three mesial canals in $13.3 \%$ of the cases [7] and two canals in the distal root are observed in $15-17 \%$ of the cases [8] but these rare cases of anatomic variability are considered exceptions, not the rule. In this clinical case, morphologic variations, presence of tertiary dentin and limited access to the operating site, generated obstacles in carrying out the treatment. The mouth opening limitation may be attributed to the trauma he sustained in lower jaw in his childhood which remained untreated due to parent's ignorance.

A radiograph produces a 2D image of a 3D object that results in the superimposition of the overlying anatomical structures making radiographs of limited value in cases with complex root canal anatomy [9]. It, therefore, becomes mandatory for the clinician to use all the available diagnostic aids to locate and debride the entire root canal system [10]. Cone Beam Computed Tomography (CBCT) is an imaging tool that gives an accurate three-dimensional (3D) view of body structure and considered as a standard diagnostic tool in dentistry but its use in endodontics is highly controversial due to high amount of radiation and cost, a patient is exposed to and therefore, conventional radiography remains the most acceptable modality for diagnosis in endodontic [11].

Various clues and methods are documented in the literature to locate hard-to-find canals [4]. Preoperatively, extra canal can be expected in the mandibular molar when there is prominent buccal cusp present which is wide mesio-distally. Intraoperatively, use of ultrasonic devices enhances the treatment success in managing endodontic cases with higher difficulty level. These devices have become increasingly more helpful in gaining access to canal orifices and removing intracanal obstructions [12]. Utilization of diamond- coated ultrasonic tips made all the canal isthmus very clean and highly visible under magnification. Isthmus is a thin communicating channel between canal orifices, found in the floor of pulp chamber and may contain pulp or pulpally derived tissue and requires diligent cleaning to explore hidden canal orifices.

In this case, "Champagne bubble" test was performed effectively and successfully. The chamber was flooded with $\mathrm{NaOCl}$ and as soon as it came into contact with canal tissue, tiny bubbles were produced indicating the position of the missing canal orifice. The logic behind this test is that the $\mathrm{NaOCl}$ dissociates into its $\mathrm{Na}^{+}$and $\mathrm{Cl}^{-}$ 
ions and releases free Oxygen. A positive bubble test indicates that $\mathrm{NaOCl}$ is reacting with pulp tissue [13]. Methylene blue dye has also been recommended for disclosing location of hidden canals.

Locating and treating hard-to-find canals is challenging but mandatory for successful endodontic treatment. The dentist should show patience and must have appropriate relevant knowledge and proper illumination and magnification facilities to defeat the difficulties posed by hidden canals. Scarcity of a facility to have a CTBT was an obstacle which limits a dentist's diagnostic ability. Interpretation of CBCT is another limitation for most of the practicing dentists and for improvement in the situation, they should undertake specially designed hands-on workshops on CTBT as a part of continuing dental education.

\section{Conclusion}

For successful accomplishment of an endodontic procedure, applied knowledge of the tooth morphology, its variations and various techniques to locate a missing or hard-to-find root canal. Champagne bubble test is an easy and authentic method to employ without requiring any additional equipment.

\section{Bibliography}

1. Akhlaghi Mohammadzadeh Nahid., et al. "Root Canal Anatomy and Morphology of Mandibular First Molars in a Selected Iranian Population: An In Vitro Study". The Iranian Endodontic Journal 12.1 (2017): 87-91.

2. Wasti F., et al. "Root canal systems of the mandibular and maxillary first permanent molar teeth of South Asian Pakistanis". International Endodontic Journal 34 (2001): 263-266.

3. Chourasia Hemant Ramesh., et al. "Root canal morphology of mandibular first permanent molars in an Indian population". International Journal of Dentistry 2012 (2012): 745152.

4. Mohammadi Zahed., et al. "A Clinical Update on the Different Methods to Decrease the Occurrence of Missed Root Canals". The Iranian Endodontic Journal 11.3 (2016): 208-213.

5. Awooda Elhadi M., et al. "Five canals on mandibular first molar successfully managed by undergraduate dental student: An Educational Case Report”. Journal of Oral Research 7.4 (2018).

6. Vertucci Frank J. "Root canal anatomy of the human permanent teeth". Oral Surgery, Oral Medicine, Oral Pathology, and Oral Radiology 58.5 (1984): 589-599.
7. Goel NK., et al. "Study of roots canal configuration in mandibular first permanent molar". Journal of the Indian Society of Pedodontics and Preventive Dentistry 8 (1991): 12-14.

8. Caliskan MK., et al. "Root canal morphology of human permanent teeth in a Turkish population". The Journal of Endodontics 21.4 (1995): 200-204.

9. Holtzman L. "Root canal treatment of mandibular second premolar with four root canals: A case report". International Endodontic Journal 31 (1998): 364-366.

10. Poorni S., et al. "Canal complexity of a mandibular first molar". The Journal of Conservative Dentistry 12.1 (2009): 37-40.

11. Álvarez JM and Macho AZ. "Applications of CBCT in Endodontics". (2021).

12. Sujith Ramachandra., et al. "Microscope magnification and ultrasonic precision guidance for location and negotiation of second mesiobuccal canal: An in vivo study". Journal of International Society of Preventive and Community Dentistry 4.3 (2014): S209-S212.

13. Ruddle CJ. "Identifying root canals, Endodontic Strategies". Endodontic Practice 4.6 (2011): 56.

\section{Volume 5 Issue 10 October 2021 \\ (C) All rights are reserved by Asaad J Mirza., et al.}

